\title{
Fleeting images: rapid affect discrimination in the visual cortex
}

\author{
Markus Junghöfer ${ }^{\mathrm{a}}$, Dean Sabatinelli ${ }^{\mathrm{d}}$, Margaret M. Bradley ${ }^{\mathrm{d}}$, Harald T. Schupp ${ }^{\mathrm{b}}$, Thomas R. Elbert ${ }^{\mathrm{c}}$ \\ and Peter J. Lang ${ }^{d}$
}

\begin{abstract}
${ }^{\mathrm{a}}$ Institute for Biomagnetism and Biosignalanalysis, University of Münster, Münster, ${ }^{\mathrm{b}}$ Institutes for General Psychology, ${ }^{\mathrm{C} C l i n i c a l}$ and Neuropsychology, University of Konstanz, Konstanz, Germany and ${ }^{\mathrm{d} C e n t e r}$ for the Study of Emotion and Attention (CSEA), University of Florida, Gainesville, Florida, USA

Correspondence and requests for reprints to Dr Markus Junghöfer, PhD, Institute for Biomagnetism and Biosignalanalysis, University of Münster, Malmedyweg 15, 48149 Münster, Germany

Tel: + 4925183 56987; fax: + 4925183 56874; e-mail: markus.junghoefer@uni-muenster.de
\end{abstract}

Konstanzer Online-Publikations-System (KOPS)

URL: http://www.ub.uni-konstanz.de/kops/volltexte/2006/2134/

URN: http://nbn-resolving.de/urn:nbn:de:bsz:352-opus-21343

Converging electrophysiological and hemodynamic findings indi-
cate sensory processing of emotional pictures is preferred to that
of neutral pictures. Whereas neuroimaging studies of emotional
picture perception have employed stimulus durations lasting
several seconds, recent electrocortical investigations report early
visual cortical discrimination between emotionally arousing and
neutral picture processing. Here, we use a hybrid picture presenta-
tion paradigm covering a range of rapid presentation rates
$(0.75-6 \mathrm{~Hz})$, while visual system activity is recorded with functional magnetic resonance imaging. Results demonstrate widespread sensitivity to emotional arousal in the secondary and inferior temporal visual cortex. Furthermore, activity in the lateral inferior occipital and medial inferior temporal visual cortex revealed equivalent emotion-sensitive activation across all presentation rates. Results further support the notion that attention and perceptual processing are in part directed by underlying motivational systems. NeuroReport 17:225-229 (c) 2006 Lippincott Williams \& Wilkins.

Keywords: attention, emotion, functional magnetic resonance imaging, rapid serial visual presentation, visual cortex

\section{Introduction}

Considerable evidence now exists from electroencephalographic and neuroimaging research that emotional pictures receive preferential processing in the brain. In event-related potential (ERP) studies, when pictures varying in judged emotional impact are presented, a reliable waveform distinction is observed beginning around $350 \mathrm{~ms}$ after picture onset, often lasting several seconds. The amplitude of this late positive potential, focused over occipitoparietal areas, increases systematically with the reported emotional arousal of the eliciting picture stimulus (e.g. [1-3]). A typical picture viewing study will use exposure times in the range of 3-6s, with blank inter-picture intervals lasting $10 \mathrm{~s}$ or more. Consistent with the ERP work, and using similar picture presentation paradigms, functional magnetic resonance imaging (fMRI) studies have reported a greater blood oxygen level-dependent (BOLD) signal in the extrastriate, occipitoparietal, and inferior temporal cortex during emotionally arousing stimuli than during neutral visual stimuli (e.g. [4-6]). These BOLD effects occur in a time frame consonant with the slow-wave late positive potential observed during picture presentation. Interestingly, the brain regions of the visual BOLD effects also correspond well with the estimated neural generators for earlier ERPs recorded during the rapid serial visual presentation (RSVP) of emotional pictures (e.g. $[7,8])$.
Research in cognitive science of visual processing $[9,10]$ has shown that conceptual processing of visual images occurs with great rapidity. When pictures appear only for an instant, embedded in a rapid serial stream of images (i.e. presentation rates as high as $5-10 \mathrm{~Hz}$ ), participants reliably parse semantic information within the torrent, detecting and signaling specific target contents [11,12]. Junghöfer and colleagues [7] adapted the rapid serial visual presentation method for an ERP study of emotional perception, presenting pictures high and low in judged emotional arousal in high-speed sequences, while ERPs for each picture were recorded. Distinctly different waveforms were observed for the two picture types: emotionally arousing pictures (both pleasant and unpleasant) prompted a larger descending negative voltage between 150 and $250 \mathrm{~ms}$ following stimulus than affectively neutral pictures. Analyses of the electrode array revealed cortical sources in the right and left occipital, and right occipitoparietal areas, suggesting that very briefly presented emotional pictures also receive enhanced processing in the visual system, relative to lowarousal pictures. These data are consistent with the hypothesis of 'natural selective attention' [13] in which motivationally relevant stimuli activate the defensive or appetitive system and engage increased processing resources.

The aim of the present research is to investigate the BOLD response to rapidly presented picture stimuli across a range 
of durations, in a first attempt to delineate the threshold at which emotional picture processing might garner greater visual cortical activity than more neutral stimuli. The pictures are presented serially (without gaps) in separate 12-s blocks, judged to be of high or low emotional arousal. Four different picture presentation rates are examined: 0.75, $1.5,3.0$, and $6.0 \mathrm{~Hz}$. It is hypothesized that viewing highly arousing picture blocks, relative to neutral picture blocks, will enhance cortical activity in secondary visual areas, paralleling the fMRI findings using single picture presentation with longer (e.g. 12s) duration. Consistent with both the ERP and fMRI data, bilateral BOLD effects are anticipated in extrastriate, inferior temporal, and parietal visual cortex, with somewhat greater activation in the right hemisphere.

It is further hypothesized that a diminished picturearousal effect will be apparent in the secondary visual system as the rate of picture presentation increases. Although briefly presented picture stimuli are detectable in a fast serial stream, the content of the picture is not retained in memory beyond a certain rate $[9,10]$. Conceptual masking by successive pictures (at approximately $300 \mathrm{~ms}$ duration or $3.3 \mathrm{~Hz}$ ) appears to interrupt a final processing stage that includes the long-term storage of individual percepts. Conceptual masking may attenuate the widespread visual cortical enhancement seen in previous studies in which comparatively long picture durations and $\sim 10$-s inter-picture intervals allow unrestricted processing.

\section{Materials and methods \\ Study participants}

Participants included 14 introductory psychology students and four researchers at the University of Florida (six women; 17-32 years, mean age 23.0 years, 14 with righthand preference). All volunteers agreed to participate after reading a detailed consent form approved by the University of Florida Institutional Review Board.

\section{Experimental stimuli and design}

Six 216-s digital videos were assembled using Adobe Premiere software (Adobe, San Jose, California, USA). Each video presented a rapid series of color pictures selected from the International Affective Picture System [14] in a serial stream, without interstimulus intervals. According to normative ratings of emotional arousal, 216 high and 216 low arousal pictures were selected. Brightness, color distribution, contrast, physical complexity (JPEG file size at $90 \%$ quality) and mean spatial frequency did not statistically differ across the high and low arousal picture groups. Four videos were constructed consisting of $12 \mathrm{~s}$ blocks of high arousal pictures alternating with $12 \mathrm{~s}$ blocks of low arousal pictures, with picture durations of 167, 333, 667 , or $1500 \mathrm{~ms}$. All videos included equal numbers of pleasant and unpleasant pictures. The fifth video presented alternating $12 \mathrm{~s}$ blocks of low arousal pictures $(333 \mathrm{~ms}$ duration) with $12 \mathrm{~s}$ blocks of dark screen fixation-only periods, and the last video alternated blocks of $333 \mathrm{~ms}$ high arousal pictures with fixation-only periods. All videos were preceded by a $12 \mathrm{~s}$ block of practice pictures (at the corresponding duration), not included in the functional analyses. Presentation order of the videos was counterbalanced across participants. Videos were rear-projected on a screen located at the participant's feet, visible via a coilmounted mirror. A red laser pointer dot illuminated the center of the screen and served as a fixation point throughout the session.

\section{Procedure and apparatus}

The data were acquired with a 3-T GE Signa scanner (General Electric Company, Fairfield, Connecticut, USA). The scanning sequence began with the acquisition of a standard T1-weighted sagittal anatomical magnetic resonance imaging. Seven T1-weighted coronal images (in-plane matrix: $256 \times 256$; voxel size $0.7 \times 0.7 \times 6.5 \mathrm{~mm}$ ) were acquired originating $1 \mathrm{~cm}$ anterior to the occipital pole, extending through Brodmann areas 18 and 19, with the superior portion of the final image generally including substantial tissue anterior to the occipitoparietal fissure. Functional gradient-echo echoplanar T2*-weighted images were acquired in the same locations (in-plane matrix: $128 \times 128$; voxel size: $1.41 \times 1.41 \times 6.5 \mathrm{~mm}$, TE $40 \mathrm{~ms}$, TR $3 \mathrm{~s}, \mathrm{FA} 90$ ). Functional images were collected continuously during each video, resulting in 72 volumes per video.

\section{Data reduction and analysis}

The functional images were analyzed using statistical parametric mapping [15]. Using a mutual information algorithm, the structural coronal images were coregistered with the sagittal scout, and these transformation parameters were then applied to the functional images. Images were slice-time corrected, realigned, resampled to an isotropic voxel size of $2 \times 2 \times 2 \mathrm{~mm}$, and spatially normalized to the laterality symmetric Montreal Neurological Institute (MNI) standard brain. All volumes were smoothed with an isotropic 5-mm full-width at half-maximum Gaussian kernel.

The experimental conditions were modeled as a boxcar function with nine cycles of eight scans each, convolved with an approximated hemodynamic response function. Condition effects were estimated according to the general linear model, and specific effects were compared using linear contrasts. Across-subject random effects analysis was performed on contrasts of interest. Across-subject laterality effects were conducted with paired $t$-tests, testing the null hypothesis that the participants' mean of a specific normalized contrast image voxel is identical to its laterally (leftright) mirrored mean contrast voxel. Although spatial normalization of individual functional scans has been performed using a left-right symmetric standard target image, it has to be noted that suboptimal normalization leaving residual structural asymmetry could result in artificial effects of functional hemispheric dominance. Anatomical MNI localizations were transformed to Talairach space on the basis of [16].

\section{Results}

Consistent with past work, a contrast of BOLD signal between all high and low arousal picture blocks revealed greater signal change during emotionally arousing picture perception in widespread areas of visual cortex. These structures include the extrastriate occipital, medial parietal, and inferior temporal cortex (see Table 1). A contrast of BOLD signal changes as a function of emotional arousal between the hemispheres demonstrated significantly greater 
Table I Location and cluster sizes of significant blood oxygen level-dependent difference activations for specific contrasts of interest

\begin{tabular}{|c|c|c|c|c|}
\hline Contrast & Structure & L/R coordinates $(x, y, z)$ & $\mathrm{L} / \mathrm{R}$ peak $t$ value & L/R cluster size \\
\hline \multirow{7}{*}{$\begin{array}{l}\text { High > low (arousal effect; } \\
\text { all presentation rates) }\end{array}$} & Middle occ. gyrus, BA 19 & $-42-72-3 / 46-779$ & $7.5 / 11.2$ & $604 / 942$ \\
\hline & Precuneus, BA 7 & $-28-6435 / 22-6638$ & $5.9 / 6.0$ & $542 / 677$ \\
\hline & Middle temp. gyrus, BA 39 & $-46-66 I I / 46-759$ & $9.5 / 10.4$ & $209 / 373$ \\
\hline & Inferior occ. gyrus, BA I8 & $-44-740 / 44-78 I$ & $6.2 / 8.1$ & $288 / 274$ \\
\hline & Middle occ. gyrus, BA 37 & $-46-649 / 48-724$ & $9.6 / 9.1$ & $119 / 183$ \\
\hline & Precuneus, BA 31 & $-26-7828 / 22-7630$ & $2.7 / 4.3$ & $30 / 45$ \\
\hline & Lingual gyrus, BA I7 & $-14-88-2 / 18-87 \mid$ & $3.2 / 4.8$ & $30 / 34$ \\
\hline Right > left (arousal effect; & Middle occ. gyrus, BA 19 & $144-764$ & /4.4 & $/ 246$ \\
\hline \multirow[t]{3}{*}{ all presentation rates) } & Precuneus, BA 7 & $20-6633$ & 3.5 & $/ 134$ \\
\hline & Middle occ. gyrus, BA 37 & $146-660$ & 16.9 & $/ 106$ \\
\hline & Inferior par. lobule, BA 39 & $-38-5732 / 50-6238$ & $3.2 / 3.7$ & $82 / 63$ \\
\hline \multirow[t]{2}{*}{ Fast $>$ slow (arousal effect) } & Inferior par. lobule, BA 40 & $-40-5843 / 51-5838$ & $2.8 / 3.1$ & $13 / 15$ \\
\hline & Middle occ. gyrus, BA 19 & $-28-8022 / 34-7324$ & $6.6 / 7.1$ & $185 / 285$ \\
\hline \multirow[t]{4}{*}{ Slow $>$ fast (arousal effect) } & Cuneus, BA 18 & $-26-76-6 / 24-7719$ & $3.0 / 4.0$ & $192 / 232$ \\
\hline & Precuneus, BA 7 & $-6-5452 / 22-5849$ & $4.1 / 4.3$ & $564 / 119$ \\
\hline & Precuneus, BA 3I & $-8-7228 / 24-7518$ & $3.3 / 3.7$ & $163 / 94$ \\
\hline & Cuneus, BA I7 & $-2-8312 / 20-7515$ & $6.0 / 3.6$ & $6 / 57$ \\
\hline
\end{tabular}

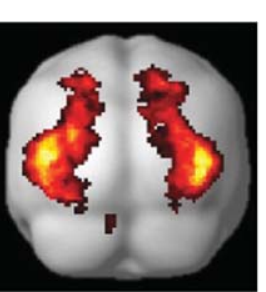

$0.75 \mathrm{~Hz}$

$(1333 \mathrm{~ms})$

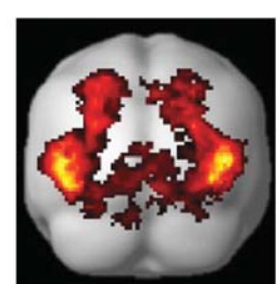

$1.5 \mathrm{~Hz}$

(667 ms)

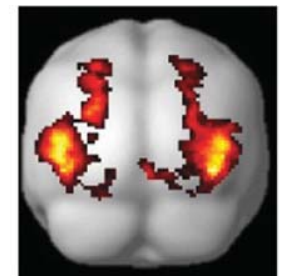

$3.0 \mathrm{~Hz}$

(333 ms)

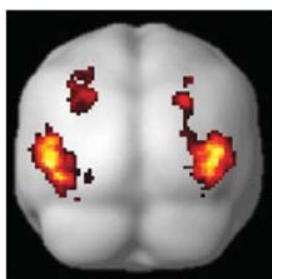

$6.0 \mathrm{~Hz}$

(167 ms)

Fig. I Random effects analysis $(P<0.05)$ of emotionally arousing versus neutral picture processing in the visual cortex. A contrast map is shown for each picture presentation rate, increasing from left to right. As presentation rate increases, the difference between blood oxygen level-dependent signal during emotional picture blocks and neutral picture blocks decreases in parietal precuneus and middle occipital gyrus, while activity in lateral inferior occipital gyrus remains constant (see Results).

activity in the right precuneus and right middle occipital gyrus than that in the same structures in the left hemisphere (Table 1).

To assess the effect of picture presentation rate on the emotion-driven BOLD signal effects, analysis of the linear effects of presentation rate revealed significantly less BOLD signal difference in several visual cortical structures as picture presentation rate increased (Table 1). These areas include the precuneus, cuneus and middle occipital gyrus. In contrast, the lateral inferior occipital gyrus and medial inferior temporal cortex were not sensitive to presentation rate: equivalent levels of BOLD signal change differentiated high from low arousal picture viewing irrespective of picture duration (see Fig. 1, Table 1). Interestingly, small clusters in the lateral inferior parietal lobule showed increasing BOLD signal difference with increasing presentation rate.

\section{Discussion}

Replicating previous work, passive viewing of affectively arousing pictures led to greater activation of the visual cortex than did the viewing of pictures rated low in emotional arousal. This emotion effect was evident in the secondary occipital, medial parietal precuneus, and inferior temporal cortex, consistent with previous fMRI studies of affective picture perception (e.g. $[5,6,17]$ ). These effects were evident despite rapid serial presentation of pictures, which did not provide any darkscreen 'off' periods between picture presentations. The cortical distribution of the arousal-driven BOLD signal increases elicited by emotionally arousing pictures is remarkably consistent with the topography of electrocortical negativity recorded at the scalp during passive viewing of rapidly presented emotional pictures, with both pointing to the involvement of the bilateral secondary occipital and medial parietal cortex $[7,8]$. This is noteworthy considering the substantial differences in temporal and spatial resolution of the two methodologies.

The signal-enhancing effects of emotional pictures in the lateral occipital cortex and portions of the medial inferior temporal visual cortex were virtually unaffected by picture presentation rate. In other words, whether each picture in a block remained on the screen for $1333 \mathrm{~ms}$, or just $167 \mathrm{~ms}$, an equivalent degree of signal increase is elicited during emotionally arousing picture blocks compared with low arousal picture blocks. In contrast, as presentation rate increased, the medial parietal 
precuneus showed decreasing (but still evident) activation enhancement during emotionally arousing picture blocks compared with low arousal picture blocks (Fig. 1). In the primary visual system, increasing visual flicker results in increasing BOLD signal change peaking at $8 \mathrm{~Hz}$ (e.g. [18]); thus, any decrement in the emotional arousaldriven BOLD contrast in the current data as rate increases may be attributed to limitations of complex stimulus processing and not to an arousal-independent BOLD signal decrease.

Small bilateral clusters associated with emotion-driven BOLD signal changes in the lateral inferior parietal lobe showed the opposite effect, increasing in activity with presentation rate, perhaps indexing increasing demands on spatial attention [19]. Models of visual memory would predict that at the fastest rate of presentation used here $(6 \mathrm{~Hz}$ or $167 \mathrm{~ms})$, long-term memory consolidation of individual stimuli would be prevented by 'conceptual masking' by successive pictures $[9,10]$, whereas immediate categorization should remain intact. The current data support this perspective, in that evidence of stimulus discrimination as a function of motivational relevance (i.e. emotional arousing versus unarousing pictures) is evident in enhanced secondary visual cortical activation. Owing to the limited temporal resolution of BOLD imaging, and the resulting requirement to block each picture series according to emotional arousal, this interpretation must be tempered by the possibility that individual pictures may 'pop out' of the rapid stream and remain in short-term memory, despite the conceptual masking of following pictures. Data from several ERP studies using rapid visual presentation of affective pictures, however, demonstrate that secondary visual areas reliably differentiate these contents, lending weight to the argument that these effects are not the result of an occasional, highly salient picture.

Although all active visual areas showed a strong bilateral pattern, a bias toward the right hemisphere was evident in the contrast between emotionally arousing and unarousing pictures - also replicating prior fMRI and ERP studies of picture processing $[5,7,17,20,21]$. These results are consistent with neurological and neurophysiological findings $[22,23]$ proposing that emotionally arousing visual stimuli might be associated with greater right hemisphere and posterior parietal activation. No differences in rate sensitivity were seen across the hemispheres; thus, the picture processing and arousal effects appear to be consistently skewed to the right, but do not interact with presentation rate.

\section{Conclusion}

We have suggested that in the absence of instructions, attention is modulated by activity in the brain's motivational systems, such that cues that prime appetitive or defensive circuitry automatically trigger orienting to the stimulus for further processing [13]. This idea is well supported in the animal literature, in which subcortical structures have been demonstrated to be intimately involved in directing attention to motivationally relevant stimuli [24-26]. The current data support the idea that emotionally arousing pictures not only engender enhanced sensory processing, but can also do so with very brief presentation, suggesting that survival relevant stimuli are preferentially evaluated and may be acted upon without extensive processing.

\section{References}

1. Cacioppo JT, Crites SL Jr, Gardner WL, Bernston GG. Bioelectrical echoes from evaluative categorizations: I. A late positive brain potential that varies as a function of trait negativity and extremity. J Pers Soc Psychol 1994; 67:115-125.

2. Cuthbert BN, Schupp HT, Bradley MM, Birbaumer N, Lang PJ. Brain potentials in affective picture processing: covariation with autonomic arousal and affective report. Biol Psychol 2000; 52:95-111.

3. Schupp HT, Cuthbert BN, Bradley MM, Cacioppo JT, Ito T, Lang PJ Affective picture processing: the late positive potential is modulated by motivational relevance. Psychophysiology 2000; 37:257-261.

4. Breiter HC, Etcoff NL, Whalen PJ, Kennedy WA, Rauch SL, Buckner R, et al. Response and habituation of the human amygdala during visual processing of facial expression. Neuron 1996; 17:1-13.

5. Bradley MM, Sabatinelli D, Lang PJ, Fitzsimmons JR, King WM, Desai P. Activation of the visual cortex in motivated attention. Behav Neurosci 2003; 117:369-380.

6. Sabatinelli D, Flaisch T, Bradley MM, Fitzsimmons JR, Lang PJ. Affective picture perception: Gender differences in visual cortex? Neuroreport 2004; 15:1109-1112.

7. Junghöfer M, Bradley MM, Elbert TR, Lang PJ. Fleeting images: a new look at early emotion discrimination. Psychophysiology 2001; 38: 175-178.

8. Schupp H, Junghöfer M, Weike AI, Hamm AH. Attention and emotion an ERP analysis of facilitated emotional stimulus processing. Neuroreport 2003; 14:1107-1110.

9. Intraub $\mathrm{H}$. Understanding and remembering briefly glimpsed pictures: Implications for visual scanning and memory. In: Coltheart V, editor. Fleeting memories: cognition of brief visual stimuli. Cambridge, Massachusetts: The MIT Press; 1999. pp. 47-70.

10. Potter MC, Staub A, Rado J, O'Connor DH. Recognition memory' for briefly presented pictures: the time course of rapid forgetting. J Exp Psychol Hum Percept Perform 2002; 28:1163-1175.

11. Thorpe S, Fize D, Marlot C. Speed of processing in the human visual system. Nature 1996; 381:520-522.

12. Keysers C, Xiao OK, Foldiak P, Perrett DI. The speed of sight. J Cogn Neurosci 2001; 13:90-101.

13. Lang PJ, Bradley MM, Cuthbert BN. Motivated attention: affect, activation and action. In: Lang PJ, Simons RF, Balaban MT, editors. Attention and orienting: sensory and motivational processes. Hillsdale, New Jersey: Erlbaum; 1997. pp. 97-136.

14. Lang PJ, Bradley MM, Cuthbert BN. International Affective Picture System (IAPS): technical manual and affective ratings. Gainesville, Florida: The Center for Research in Psychophysiology; 1999.

15. Friston KJ, Holmes AP, Worsley KJ, Poline JB, Frith CD, Frackowiak RSJ. Statistical parametric maps in functional imaging: a general linear approach. Hum Brain Mapp 1995; 2:189-210.

16. Lancaster JL, Woldorff MG, Parsons LM, Liotti M, Freitas CS, Rainey L, et al. Automated Talairach atlas labels for functional brain mapping. Hum Brain Mapp 2000; 10:120-131.

17. Lang PJ, Bradley MM, Fitzsimmons JR, Cuthbert BN, Scott JD, Moulder B, et al. Emotional arousal and activation of the visual cortex: an fMRI analysis. Psychophysiology 1998; 35:199-210.

18. Thomas CG, Menon RS. Amplitude response and stimulus presentation frequency response of human primary visual cortex using BOLD EPI at 4T. Magn Res Med 1998; 40:203-209.

19. Corbetta M, Shulman GL. Control of goal-directed and stimulus-driven attention in the brain. Nat Rev Neurosci 2002; 3:201-215.

20. Keil A, Moratti S, Sabatinelli D, Bradley MM, Lang PJ. Additive effects of emotional content and spatial selective attention on electrocortical facilitation. Cereb Cortex 2005; 15:1187-1197.

21. Pizzagalli D, Regard M, Lehmarm D. Rapid emotional face processing in the human right and left brain hemispheres: an ERP study. Neuroreport 1999; 10:1-8.

22. Heilman KM, Watson RT, Bowers D. Affective disorders associated with hemispheric disease. In: Heilman KM, Satz P, editors. Neuropsychology of human emotion. New York: Guilford; 1983. pp. 45-64. 
23. Heller W. The neuropsychology of emotion. In: Stein N, Leventhal B, Trabasso T, editors. Psychological and biological approaches to emotion. Hillsdale, New Jersey: Erlbaum; 1990.

24. Cardinal RN, Parkinson JA, Hall J, Everitt BJ. Emotion and motivation: the role of the amygdala, ventral striatum, and prefrontal cortex. Neurosci Biobehav Rev 2002; 26:321-352.
25. Davis M, Whalen PJ. The amygdala: vigilance and emotion. Mol Psychiatry 2001; 6:13-34.

26. Holland PC, Han JS, Gallagher M. Lesions of the amygdala central nucleus alter performance on a selective attention task. J Neurosci 2000; 20:6701-6706. 